\title{
Morfometria do Trato Digestório da Tartaruga-da-amazônia (Podocnemis expansa) Criada em Sistema Comercial
}

\author{
Vera Lúcia Ferreira Luz ${ }^{1}$, José Henrique Stringhini², Yeda Soares de Lucena Bataus ${ }^{3}$, Wesley \\ Assis de Paula ${ }^{4}$, Michel Neto Novais ${ }^{4}$, Isaías José dos Reis ${ }^{5}$
}

\begin{abstract}
RESUMO - Estudos de parâmetros morfométricos do trato gastrointestinal (TGI) são necessários para o conhecimento dos processos digestórios dos alimentos no organismo animal e indicar a preferência alimentar de uma espécie. Foram amostrados, bimensalmente, 100 filhotes capturados aleatoriamente em oito criadouros no município de Diorama, Goiás. O experimento foi conduzido com animais entre 23 a 29 meses de idade, que tiveram seus desempenhos avaliados por medidas biométricas do comprimento retilíneo da carapaça e do peso. Para o cálculo das relações corporais dos órgãos tomou-se como base o peso vivo individual de cinco tartarugas, em cada idade estudada, perfazendo um total de quatro colheitas com 20 exemplares de cada criadouro. As análises de comparação de médias foram realizadas pelo teste de Duncan. Os resultados indicaram que o estômago representou maior percentual do trato digestório, com 44,20\%, seguido pelo intestino delgado, $28,48 \%$, e o intestino grosso, com 20,93\%, baseados na relação corporal com o TGI vazio. O TGI cheio apresentou comprimento médio de $72,75 \mathrm{~cm}$. Para o intestino delgado foi obtida a média de $46,68 \mathrm{~cm}$ e para o intestino grosso 14,00 cm. As análises das relações corporais indicaram que o estômago e o intestino delgado apresentaram maior capacidade de armazenamento, sugerindo que desempenham importante função na digestão de alimentos consumidos.
\end{abstract}

Palavras-chave: Podocnemis expansa, morfometria, manejo em cativeiro

\section{Digestive Tract Morphometric of Tartaruga-da-Amazônia (Podocnemis expansa) Kept in Commercial Captive Systems}

\begin{abstract}
It is necessary to carry out a study that focuses on the morphometrical parameters of the gastrointestinal tract (TGI), which can provide subsidies for getting knowledge about the digestive processes that happen into the animal organism. Bimontly, it was sampled 100 P. expansa hatchlings, which were randomly captured from eight commercial flocks located at the county of Diorama, State of Goiás/Brazil. The experiment was conducted with animals of 23 to 29 months of ages, from which the biometrical measures were taken, in order to evaluate their growth performances. It was taken two biometrical measures, that is, the carapace rectlineal length - in milimeters, and the weight gain - in grams. Aiming at the calculation of the body relationships, it was taken, as a basis, the individual gross weight of five hatchlings, from each one of the studied ages, totalizing an amount of four crops of 20 specimens from each involved commercial flock. The comparison analyses of the averages were accomplished by Duncan test. Based upon the relations between the empty TGI and the whole body, the results pointed out that the stomach corresponded to the major portion of the digestive tract, that reached $44.20 \%$ of it. Second, came the small gut, that reached $28.48 \%$ of it, followed by the large gut, that reached $20.93 \%$ of the digestive tract. The full TGI showed a mean lenght of $72.75 \mathrm{~cm}$. As for the small gut, it was obtained an average length of $46.68 \mathrm{~cm}$ and, as for the large gut, it was observed an average lenght of $14.00 \mathrm{~cm}$. The body relation analyses indicated that the stomach and the small gut showed the best storage capacities and, this result suggests that those viscera play an important role in the food digestion.
\end{abstract}

Key Words: Podocnemis expansa, morphometrics, management in captivity

\section{Introdução}

A possibilidade de criação comercial da Podocnemis expansa, espécie conhecida como Tartaruga-daamazônia, é resultado dos trabalhos de sua proteção e manejo na natureza e tem-se mostrado como investi- mento promissor, não só pelo aspecto econômico, como pela relevância que assume na cultura amazônica.

Dentre as espécies de quelônios amazônicos a tartaruga-da-amazônia está legalmente liberada à criação comercial, pelo seu potencial para a exploração zootécnica. Com isso, o desenvolvimento de

\footnotetext{
1 Parte da dissertação de mestrado em Medicina Veterinária apresentada à UFG pelo primeiro autor. Médica Veterinária do RAN/IBAMA/GO E.mail: vera.luz@ibama.gov.br

2 Engenheiro-Agrônomo, Professor do Departamento de Produção Animal da EV/UFG. E.mail: henrique@vet.ufg.br

${ }^{3}$ Engenheira Florestal do RAN/IBAMA/GO. E.mail: yeda@go.ibama.gov.br

${ }^{4}$ Acadêmico de Medicina Veterinária/UFG.

5 Biólogo do RAN/IBAMA/GO. E.mail: isaias.reis@ibama.gov.br
} 
sistemas de criação pode contribuir para a diminuição da pressão sobre os animais de vida livre (CENAQUA, 2000).

$\mathrm{O}$ interesse pela criação comercial da tartaruga tem crescido nas Regiões Norte e Centro-Oeste, com um total de 98 criadouros registrados até o momento. Dentre estes, 27 possuem animais para serem comercializados. Dentro da política desenvolvida na criação, estabelece-se como padrão os animais que atingirem o peso de $1,5 \mathrm{~kg}$ em um prazo de dois anos (RAN, 2001).

Sobre o hábito alimentar de quelônios na natureza, Almeida et al. (1986), Terán (1992) e Terán et al. (1995) observaram que a alimentação da tartaruga-daamazônia constituiu-se de frutos, raízes, sementes e talos de folhas de plantas de várzeas, sugerindo que os vegetais representam $97 \%$ da sua alimentação.

Em cativeiro, a tartaruga requer uma infra-estrutura semelhante àquela da piscicultura. Alfinito (1980) sugeriu como fundamental para sua criação, a partir de filhotes recém-nascidos, a instalação de berçários com rampas e comedouros, manutenção permanente de um curso d'água e alimentação. Elaborou, ainda, uma lista de plantas nativas, assinalando certos moluscos e pequenos crustáceos como alimento. Adicionalmente, informou que, na natureza, o animal é considerado herbívoro e em cativeiro apreende qualquer tipo de alimento.

Lima (1998) avaliou o efeito de dietas variando a fonte de proteína oferecida (origem animal e vegetal) em filhotes de $P$. expansa pós-eclosão até doze meses de idade. Cinco dietas com diferentes fontes de proteína na matéria seca $(100 \%$ vegetal; $75 \%$ vegetal e $25 \%$ animal; $50 \%$ animal e $50 \%$ vegetal; $25 \%$ vegetal e $75 \%$ animal; $100 \%$ animal) foram fornecidas a mil exemplares que tiveram seu desempenho avaliado por medidas biométricas, parâmetros hematológicos e metabólicos plasmáticos. A ração com $50 \%$ de proteína animal e $50 \%$ vegetal foi a que proporcionou maior ganho médio de peso final $(512,79$ $\pm 12,48 \mathrm{~g}$ ) e melhor homeostase fisiológica, sendo a que apresentou a melhor composição protéica para a criação de tartaruga em cativeiro.

As características anatômicas, morfológicas e histológicas do trato digestório de $P$. expansa jovens e recém-eclodidas foram registradas pelo CENAQUA (1992) e por Oliveira et al. (1996); e em adultas, por Santos et al. (1998). Estes últimos autores verificaram que o esôfago apresentou a mucosa pregueada revestida por um epitélio estratificado prismático e não possui glândulas esofágicas. O estômago apresentou uma forma achatada, com a região pilórica bem desenvolvida e encurvada, contendo glândulas gástricas com células secretoras, além de células acidófilas nas glândulas pilóricas. O intestino delgado é longo e bem enovelado, visto que sua mucosa possui inúmeras vilosidades e seu epitélio é simples e prismático com borda estriada e células caliciformes. O intestino grosso iniciou com uma dilatação do cólon, este bem curto, e a mucosa com muitas vilosidades é revestida por um epitélio pseudo-estratificado prismático com células caliciformes em grande quantidade. A cloaca apresentou mucosa com inúmeras pregas constituindo um relevo complexo.

De acordo com o RAN (2001), a principal dificuldade enfrentada pela quelonicultura é a questão alimentar. Pouco se conhece sobre as reais exigências nutricionais da tartaruga. Estudos têm indicado que $90 \%$ da alimentação da tartaruga, em condições naturais, é composta de vegetais. O item alimentar mais utilizado na criação de tartaruga tem-se constituído de ração para peixes, com níveis protéicos variando de $28 \%$ a $30 \%$ de proteína bruta, e é considerado o melhor alimento disponível no mercado. A compreensão de mecanismos digestórios é de fundamental importância para o desenvolvimento e aprimoramento de sistemas de arraçoamento. Estudos de parâmetros morfométricos do trato gastrointestinal (TGI) se fazem necessários para fornecer subsídios sobre os processos digestórios dos alimentos no organismo animal e indicar a preferência alimentar de uma espécie. Portanto, esse trabalho teve como objetivo quantificar os parâmetros morfométricos dos órgãos do trato digestório de exemplares de Podocnemis expansa, com idades entre 23 e 29 meses, mantidos em criadouros comerciais.

\section{Material e Métodos}

O experimento foi conduzido no período de novembro de 1999 a julho de 2000 e a primeira etapa foi realizada no município de Diorama, Goiás, localizado na região oeste do Estado, onde se acompanhou o crescimento de Podocnemis expansa em oito criadouros comerciais, licenciados pelo Ibama. $\mathrm{Na}$ segunda etapa, realizou-se a avaliação dos parâmetros morfométricos do trato digestório. Essa fase foi conduzida no Laboratório de Medicina Veterinária Preventiva da Escola de Veterinária da Universidade Federal de Goiás. 
A colheita dos dados biométricos foi conduzida no período de 15 de novembro de 1999 a 22 de maio de 2000 , correspondendo ao intervalo entre o $23^{\circ}$ e o $29^{\circ}$ meses de idade. Foi tomada uma amostra mensal, aleatória, de 100 animais, capturados por rede de arrasto e retiradas suas medidas biométricas. Os procedimentos para realização das medidas biométricas foram aqueles utilizados pelo CENAQUA (1999). Foi medido o comprimento retilíneo da carapaça, em milímetro, com o auxílio de paquímetro de metal, com capacidade de $200 \mathrm{~mm}$, além de paquímetro de madeira, com capacidade de $50 \mathrm{~cm}$ e precisão de $1 \mathrm{~cm}$, conforme o estágio de crescimento do animal. As medidas foram tomadas entre a extremidade anterior do contato dos primeiros escudos marginais até o ponto mais posterior da sutura dos escudos supracaudais (Figura 1). O peso foi obtido utilizando-se balança eletrônica com capacidade de $3 \mathrm{~kg}$ e precisão de $1 \mathrm{~g}$, e calculados o ganho de peso e o comprimento da carapaça nos diferentes períodos.

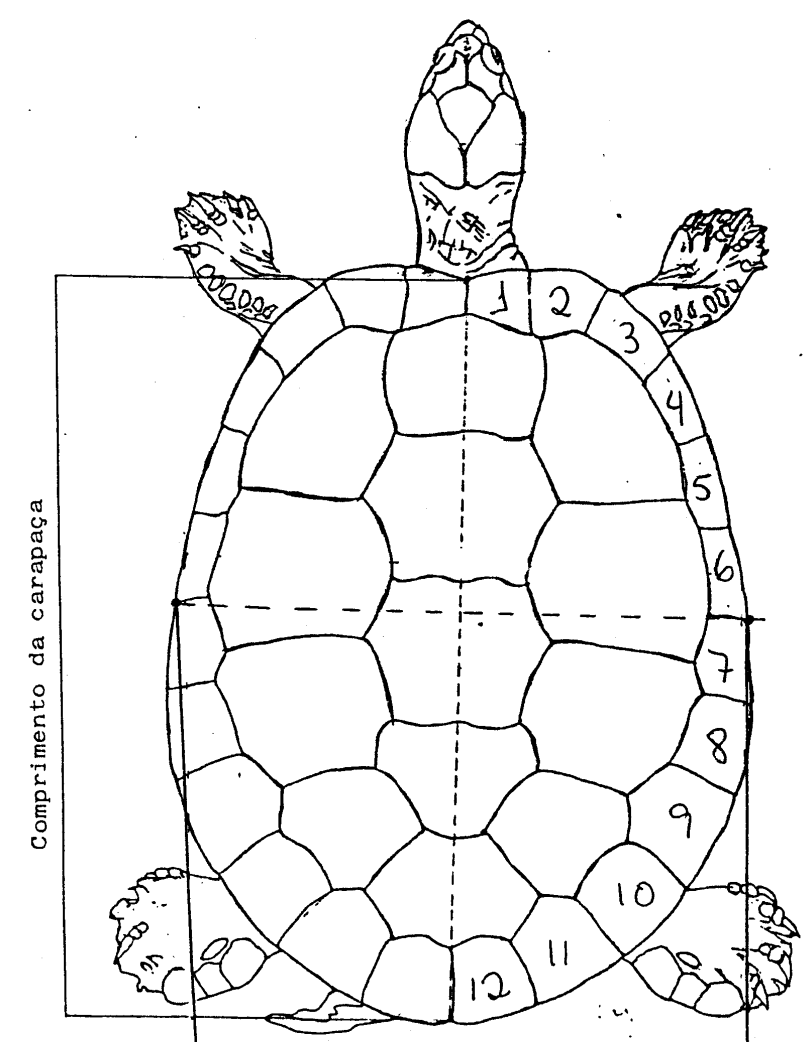

Figura 1 - Representação da medida biométrica do comprimento retilíneo da carapaça em Podocnemis expansa (tartaruga-da-amazônia).

Figure 1 - Rectilineal biometric measure of the carapace length of Podocnemis expansa (tartatuga-daamazônia).
Para obtenção dos dados e cálculos sobre a morfometria do trato digestório foram selecionadas cinco tartarugas, de cada criadouro, com pesos próximos aos valores médios obtidos, registrados nas biometrias do $23^{\circ}$, $25^{\circ}$, $27^{\circ}$ e $29^{\circ}$ meses de idade, perfazendo um total de quatro colheitas com 20 exemplares de cada criadouro. Procedeu-se à pesagem dos animais e mediu-se o comprimento retilíneo da carapaça, em milímetros. Em seguida, eles foram identificados e transportados para o Departamento de Medicina Veterinária Preventiva da Escola de Veterinária da UFG/Goiânia - GO, visando ao abate e à avaliação dos parâmetros morfométricos do trato digestório.

O abate foi realizado conforme os procedimentos sugeridos por Silva Neto (1998). Para insensibilização dos animais, estes foram colocados em um recipiente, contendo água e gelo a uma temperatura em torno de $5^{\circ} \mathrm{C}$, durante 20 minutos. Para o abate foi realizada a secção da cabeça. Após a retirada do plastrão com auxílio de uma serra giratória elétrica, foi efetuada manualmente a retirada das vísceras do trato digestório, fígado, pâncreas, gordura, baço, pulmões, aparelho excretor e órgãos reprodutores.

Para o cálculo de rendimento dos órgãos, tomou-se como base o peso vivo individual de cinco tartarugas, em cada idade estudada. As variáveis foram analisadas para cada idade e os valores obtidos foram tabulados e apresentados em porcentagem para o peso do trato gastrointestinal (TGI) vazio (esôfago, estômago, intestino delgado e intestino grosso). O comprimento total do TGI, do intestino delgado e do intestino grosso foi analisado em função dos valores obtidos, individualmente, com régua graduada em centímetros e precisão de 1 milímetro. A relação corporal com o TGI vazio nos diversos segmentos do trato digestório foi determinada com base nos estudos de Andrade (2000), de acordo com os seguintes cálculos:

$$
\begin{aligned}
& \frac{\text { PEV }}{\text { PTGIV }} \times 100 \\
& \frac{\text { PIDV }}{\text { PTGIV }} \times 100 \\
& \frac{\text { PIGV }}{\text { PTGIV }} \times 100
\end{aligned}
$$

sendo $\mathrm{RCE}=$ relação corporal para o estômago; $\mathrm{PEV}=$ peso do estômago vazio; PTGIV = peso do TGI vazio; RCID = relação corporal para o intestino delgado;

R. Bras. Zootec., v.32, n.1, p.10-18, 2003 
PIDV = peso do intestino delgado vazio; $\mathrm{RCIG}=$ relação corporal para o intestino grosso; PIGV = peso do intestino grosso vazio.

A capacidade de armazenamento do estômago (CAE) foi definida como sendo o percentual da razão entre o bolo alimentar do estômago e o alimento digerido no TGI, conforme os cálculos abaixo:

$$
\frac{\text { PEC - PEV }}{\text { PTGIC - PTGIV }} \times 100
$$

sendo: $\mathrm{PEC}=$ peso do estômago cheio $\mathrm{PEV}=$ peso do estômago vazio; PTGIC = peso do TGI cheio; PTGIV $=$ peso do TGI vazio.

As análises estatísticas foram realizadas de acordo com o recomendado por Sampaio (1998), utilizando-se o programa ESTAT (Sistema de Análises Estatísticas) desenvolvido pelo Departamento de Ciências Exatas da Universidade Estadual Paulista/ Faculdade de Ciências Agrárias e Veterinárias, Campus de Jaboticabal. Foi aplicado o Teste de Duncan a 5\% de probabilidade para comparação de médias. Para tanto, os dados porcentuais do peso do trato gastrointestinal vazio, peso do intestino delgado vazio, peso do intestino grosso vazio e capacidade de armazenamento do estômago foram transformados em arco seno $v x$

\section{Resultados e Discussão}

Analisadas as relações corporais do trato digestório (TGI) de P. expansa nos oito criadouros, entre 23 e 29 meses de idade, comparados pelo Teste de Duncan, observou-se que a relação trato gastrointestinal (TGI) vazio e peso corporal médio (Tabela 1) não apresentou diferença $(\mathrm{P}>0,05)$ para esses indivíduos em todos os criadouros. Excetua-se o $27^{\circ}$ mês, quando se obtiveram diferenças porcentuais entre o criadouro Campo Redondo, com maior porcentual $(4,29 \%)$, e o Alcantilhado, com menor $(1,47 \%)$, de acordo com os valores médios do comprimento da carapaça e do peso corporal, entre o $23^{\circ} \mathrm{e}$ $29^{\circ}$ mês de idade (Tabela 2).

As variáveis morfométricas do estômago, intestinos delgado e grosso vazios, baseadas na relação corporal com o TGI vazio, nos diferentes períodos, estão expressas nas Tabelas 3 e 4 . Os resultados demonstraram que o estômago representou maior porcentual do trato digestório, com $44,20 \%$, seguido pelo intestino delgado, que correspondeu a $28,68 \%$, e pelo intestino grosso, a 20,93\%. Em todos os períodos,

Tabela 1 - Porcentual do trato gastrointestinal (TGI) vazio, em relação ao peso corporal médio de Podocnemis expansa, com idade entre 23 e 29 meses, procedentes de criadouros comerciais $(n=5)$

Table 1 - Percentage of the empty gastrointestinal tract, in relation to the whole body weight of P. expansa from 23 to 29 months, from commercial flocks $(n=5)$

\begin{tabular}{lcccc}
\hline $\begin{array}{l}\text { Criadouros } \\
\text { Commercial flocks }\end{array}$ & \multicolumn{3}{c}{$\begin{array}{c}\text { Idade (meses) } \\
\text { Age (months) }\end{array}$} \\
\cline { 2 - 5 } & 23 & 25 & 27 \\
\hline & & & TGIvazio(\%) \\
Empty TGI (\%) & \\
Faz. Vale da Serra & $3,78^{\mathrm{a}}$ & $3,48^{\mathrm{a}}$ & $3,15^{\mathrm{b}}$ & $3,01^{\mathrm{a}}$ \\
Pró-Fauna & $3,61^{\mathrm{a}}$ & $3,88^{\mathrm{a}}$ & $3,88^{\mathrm{ab}}$ & $3,36^{\mathrm{a}}$ \\
Agrotec & $3,56^{\mathrm{a}}$ & $3,85^{\mathrm{a}}$ & $3,62^{\mathrm{ab}}$ & $3,43^{\mathrm{a}}$ \\
Faz. São Romão & $3,17^{\mathrm{a}}$ & $3,57^{\mathrm{a}}$ & $3,21^{\mathrm{b}}$ & $3,54^{\mathrm{a}}$ \\
Faz. Campo Redondo & $3,84^{\mathrm{a}}$ & $4,11^{\mathrm{a}}$ & $4,29^{\mathrm{a}}$ & $3,51^{\mathrm{a}}$ \\
Faz. Lambari & $3,62^{\mathrm{a}}$ & $3,70^{\mathrm{a}}$ & $3,21^{\mathrm{b}}$ & $2,94^{\mathrm{a}}$ \\
Faz. Rios dos Bois & $3,82^{\mathrm{a}}$ & $3,47^{\mathrm{a}}$ & $3,47^{\mathrm{b}}$ & $3,39^{\mathrm{a}}$ \\
Faz. Alcantilhado & $3,70^{\mathrm{a}}$ & $3,94^{\mathrm{a}}$ & $1,47^{\mathrm{c}}$ & $3,65^{\mathrm{a}}$ \\
Média (Mean) & 3,64 & 3,75 & 3,29 & 3,35 \\
CV(\%) & 9,27 & 7,65 & 4,61 & 7,28 \\
\hline
\end{tabular}

Médias seguidas por letras iguais, na mesma coluna, não diferem entre si pelo teste Duncan $(P>0,05)$; $C V=$ coeficiente de variação. Means indicated by the same letters, in the same column, do not differ by Duncan range test $(P>.05) ; C V=$ coefficient of variation. 
Tabela 2 - Valores médios do comprimento retilíneo da carapaça ( $\mathrm{mm}$ ) e peso corporal (g) de Podocnemis expansa, com idade entre 23 e 29 meses, de criadouros comerciais $(n=100)$

Table 2 - Mean value rectileneal carapace length $(\mathrm{mm})$ and body weight $(\mathrm{g})$ of Podocnemis expansa from 23 to 29 months, from comercial flocks $(n=100)$

\begin{tabular}{|c|c|c|c|c|}
\hline \multirow[t]{2}{*}{$\begin{array}{l}\text { Criadouros } \\
\text { Commercial flocks }\end{array}$} & \multicolumn{4}{|c|}{$\begin{array}{l}\text { Idade (meses) } \\
\text { Age (months) }\end{array}$} \\
\hline & 23 & 25 & 27 & 29 \\
\hline & \multicolumn{4}{|c|}{$\begin{array}{l}\text { Comprimento médio da carapaça }(\mathrm{mm}) \\
\text { Mean rectileneal carapace lenght }(\mathrm{mm})\end{array}$} \\
\hline Faz. Vale da Serra & $180,80^{\mathrm{a}}$ & $203,20^{\mathrm{a}}$ & $218,00^{\mathrm{a}}$ & $211,40^{\mathrm{a}}$ \\
\hline Pró-Fauna & $167,80^{\mathrm{bc}}$ & $179,40^{\mathrm{c}}$ & $191,20^{\mathrm{b}}$ & $188,00^{b}$ \\
\hline Agrotec & $164,20^{c}$ & $162,20^{\mathrm{d}}$ & $162,80^{\mathrm{de}}$ & $164,80^{\mathrm{d}}$ \\
\hline Faz. São Romão & $171,40^{\mathrm{b}}$ & $188,20^{\mathrm{b}}$ & $172,60^{c}$ & $175,80^{\mathrm{c}}$ \\
\hline Faz. Campo Redondo & $146,40^{\mathrm{e}}$ & $153,60^{\mathrm{e}}$ & $159,20^{\text {ef }}$ & $158,60^{\mathrm{d}}$ \\
\hline Faz. Lambari & $154,00^{\mathrm{d}}$ & $158,80^{\mathrm{de}}$ & $166,80^{\mathrm{d}}$ & $164,60^{\mathrm{d}}$ \\
\hline Faz. Rios dos Bois & $147,80^{\text {de }}$ & $146,80^{\mathrm{f}}$ & $155,20^{\mathrm{f}}$ & $147,20^{\mathrm{e}}$ \\
\hline Faz. Alcantilhado & $128,40^{\mathrm{f}}$ & $125,60^{\mathrm{g}}$ & $124,40^{\mathrm{g}}$ & $121,20^{\mathrm{f}}$ \\
\hline Média (Mean) & 157,60 & 164,72 & 167,51 & 166,45 \\
\hline \multirow[t]{2}{*}{$\mathrm{CV}(\%)$} & 3,30 & 2,73 & 2,55 & 2,96 \\
\hline & \multicolumn{4}{|c|}{$\begin{array}{l}\text { Peso corporal médio }(\mathrm{g}) \\
\text { Mean body weight }(\mathrm{g})\end{array}$} \\
\hline Faz. Vale da Serra & $774,00^{\mathrm{a}}$ & $1026,00^{\mathrm{a}}$ & $1216,75^{\mathrm{a}}$ & $1195,20^{\mathrm{a}}$ \\
\hline Pró-Fauna & $585,40^{\mathrm{b}}$ & $742,60^{\mathrm{b}}$ & $849,00^{b}$ & $799,80^{\mathrm{b}}$ \\
\hline Agrotec & $531,60^{c}$ & $529,60^{c}$ & $495,00^{\mathrm{fg}}$ & $519,20^{f}$ \\
\hline Faz. São Romão & $609,20^{\mathrm{b}}$ & $771,20^{\mathrm{b}}$ & $590,40^{e}$ & $628,20^{\mathrm{c}}$ \\
\hline Faz. Campo Redondo & $468,60^{\mathrm{d}}$ & $536,80^{c}$ & $626,20^{c}$ & $607,00^{\mathrm{d}}$ \\
\hline Faz. Lambari & $477,80^{\mathrm{d}}$ & $539,00^{\mathrm{c}}$ & $591,00^{\text {de }}$ & $565,80^{\mathrm{e}}$ \\
\hline Faz. Rios dos Bois & $434,60^{\mathrm{e}}$ & $427,00^{\mathrm{d}}$ & $481,80^{\mathrm{g}}$ & $421,80^{\mathrm{g}}$ \\
\hline Faz. Alcantilhado & $287,60^{\mathrm{f}}$ & $264,40^{\mathrm{e}}$ & $254,40^{\mathrm{h}}$ & $233,80^{\mathrm{h}}$ \\
\hline Média (Mean) & 521,10 & 604,57 & 623,23 & 621,35 \\
\hline $\mathrm{CV}(\%)$ & 4,27 & 4,56 & 3,64 & 2,21 \\
\hline
\end{tabular}

Médias seguidas por letras iguais, na mesma coluna, não diferem entre si pelo teste Duncan $(P>0,05)$; $C V=$ coeficiente de variação. Means indicated by the same letters, in the same column, do not differ by Duncan range test (P>.05); CV = coefficient of variation.

tiveram suas médias diferentes, entre os criadouros $(\mathrm{P}<0,05)$, exceto para o intestino grosso no $23^{\circ}$ e $25^{\circ}$ meses. Sobre essas variáveis, Moreira \& Loureiro (1992) apresentaram, para P. expansa com quatro anos de idade mantida em cativeiro, o porcentual de cada secção do trato digestório livre de conteúdos, em relação ao peso médio total, encontrando para o estômago $40,70 \%$; intestino delgado $32,10 \%$; e intestino grosso $18,90 \%$, confirmando que o estômago, seguido pelo intestino delgado, foram os que apresentaram maior capacidade em pesos vazios.

Os autores ressaltaram a importância de estudos sobre a fisiologia digestória, enfatizando que a capacidade relativa de cada compartimento está associada aos diferentes sistemas digestórios, tendo um ruminante uma capacidade relativa do estômago maior que as outras seções do trato, e um eqüino, por sua vez, maior capacidade relativa do ceco somado ao intestino grosso.

Os resultados do presente estudo sugerem que o estômago e o intestino delgado desempenham impor- tantes funções na digestão de alimentos em $P$. expansa jovens. Essa capacidade parece estar relacionada com o hábito alimentar da tartaruga na natureza, observado por Ojasti (1971), que analisou conteúdos estomacais de dez fêmeas adultas, encontrando $86 \%$ de frutos, $4 \%$ de folhas e talos de diversas plantas e $10 \%$ de animais invertebrados, informando que, em cativeiro, os animais jovens preferem alimento de origem animal (peixe). Almeida et al. (1986), Terán (1992) e Terán et al. (1995) encontraram como conteúdos estomacais de $P$. expansa frutos, raízes, sementes, talos de folhas, sugerindo que os vegetais compunham $97 \%$ de sua alimentação.

As capacidades de armazenamento do estômago em relação ao trato digestório cheio para $P$. expansa (Tabela 5) não apresentaram diferenças $(\mathrm{P}<0,05)$ para o $23^{\circ}$ e $29^{\circ}$ períodos estudados. O valor médio obtido de $69,65 \%$ foi semelhante ao encontrado por Moreira \& Loureiro (1992) para esse órgão (70,60\%) em relação ao peso corporal, confirmando ser este o maior 
Tabela 3 - Porcentual do peso do estômago vazio, intestino e delgado vazio em relação ao peso do trato gastrointestinal vazio de Podocnemis expansa, com idade entre 23 e 29 meses, procedentes de criadouros comerciais $(n=5)$

Table 3 - Percentage of the empty stomach and small gut, in relation to of the empty gastrointestinal tract of Podocnemis expansa from 23 to 29 months, from commercial flocks $(n=5)$

\begin{tabular}{|c|c|c|c|c|}
\hline \multirow[t]{2}{*}{$\begin{array}{l}\text { Criadouros } \\
\text { Commercial flocks }\end{array}$} & \multicolumn{4}{|c|}{$\begin{array}{l}\text { Idade (meses) } \\
\text { Age (months) }\end{array}$} \\
\hline & 23 & 25 & 27 & 29 \\
\hline & \multicolumn{4}{|c|}{$\begin{array}{l}\text { Estômago vazio (\%) } \\
\text { Empty stomach (\%) }\end{array}$} \\
\hline Faz. Vale da Serra & $46,84^{\mathrm{bc}}$ & $42,86^{\mathrm{bc}}$ & $37,85^{\mathrm{b}}$ & $41,37^{\mathrm{cd}}$ \\
\hline Pró-Fauna & $51,52^{\mathrm{ab}}$ & $39,46^{\mathrm{cd}}$ & $40,07^{\mathrm{b}}$ & $45,04^{\mathrm{bc}}$ \\
\hline Agrotec & $54,42^{\mathrm{a}}$ & $50,61^{\mathrm{a}}$ & $46,28^{\mathrm{a}}$ & $55,01^{\mathrm{a}}$ \\
\hline Faz. São Romão & $44,06^{\mathrm{c}}$ & $47,02^{\mathrm{ab}}$ & $45,73^{\mathrm{a}}$ & $49,55^{\mathrm{b}}$ \\
\hline Faz. Campo Redondo & $43,87^{\mathrm{c}}$ & $40,45^{\mathrm{cd}}$ & $37,50^{\mathrm{b}}$ & $38,53^{\mathrm{d}}$ \\
\hline Faz. Lambari & $47,09^{\mathrm{bc}}$ & $37,16^{\mathrm{d}}$ & $44,56^{\mathrm{a}}$ & $44,31^{\mathrm{bc}}$ \\
\hline Faz. Rios dos Bois & $51,46^{\mathrm{ab}}$ & $42,32^{b c}$ & $45,64^{\mathrm{a}}$ & $45,59^{\mathrm{bc}}$ \\
\hline Faz. Alcantilhado & $46,12^{\mathrm{bc}}$ & $35,87^{\mathrm{d}}$ & $38,92^{\mathrm{b}}$ & $36,55^{\mathrm{d}}$ \\
\hline Média (Mean) & 48,17 & 41,97 & 42,18 & 44,49 \\
\hline \multirow[t]{2}{*}{$\mathrm{CV}(\%)$} & 6,07 & 5,15 & 4,55 & 5,34 \\
\hline & \multicolumn{4}{|c|}{$\begin{array}{l}\text { Intestino delgado vazio }(\%) \\
\text { Empty small gut }(\%)\end{array}$} \\
\hline Faz. Vale da Serra & $34,72^{\mathrm{a}}$ & $33,02^{\mathrm{a}}$ & $32,21^{\mathrm{b}}$ & $36,72^{\mathrm{a}}$ \\
\hline Pró-Fauna & $22,84^{\mathrm{bc}}$ & $32,42^{\mathrm{a}}$ & $30,29^{\mathrm{b}}$ & $32,93^{\mathrm{a}}$ \\
\hline Agrotec & $29,27^{\mathrm{ab}}$ & $29,72^{\mathrm{a}}$ & $25,14^{\mathrm{c}}$ & $25,69^{b}$ \\
\hline Faz. São Romão & $31,12^{\mathrm{ab}}$ & $35,88^{\mathrm{a}}$ & $28,76^{\mathrm{b}}$ & $26,76^{\mathrm{b}}$ \\
\hline Faz. Campo Redondo & $30,29^{\mathrm{ab}}$ & $32,87^{\mathrm{a}}$ & $37,56^{\mathrm{a}}$ & $36,45^{\mathrm{a}}$ \\
\hline Faz. Lambari & $17,55^{\mathrm{d}}$ & $17,07^{\mathrm{b}}$ & $12,19^{d}$ & $12,49^{\mathrm{c}}$ \\
\hline Faz. Rios dos Bois & $23,21^{\mathrm{c}}$ & $30,09^{\mathrm{a}}$ & $30,88^{b}$ & $28,62^{\mathrm{b}}$ \\
\hline Faz. Alcantilhado & $29,27^{\mathrm{b}}$ & $30,06^{\mathrm{a}}$ & $30,43^{b}$ & $28,17^{\mathrm{b}}$ \\
\hline Média (Mean) & 27,76 & 30,14 & 28,33 & 28,48 \\
\hline $\mathrm{CV}(\%)$ & 7,76 & 10,86 & 4,59 & 6,72 \\
\hline
\end{tabular}

Médias seguidas por letras iguais, na mesma coluna, não diferem entre si pelo teste Duncan $(P>0,05)$; $C V=$ coeficiente de variação. Means indicated by the same letters, in the same column, do not differ by Duncan range test (P>.05); CV = coefficient of variation.

Tabela 4 - Porcentual do peso do intestino grosso vazio em relação ao peso do trato gastrointestinal vazio de Podocnemis expansa, com idade entre 23 e 29 meses, procedentes de criadouros comerciais $(n=5)$

Table 4 - Percentage of the empty large gut in relation to the whole empty gastrointestinal tract of Podocnemis expansa, from 23 to 29 months, from commercial flocks

\begin{tabular}{lcccc}
\hline $\begin{array}{l}\text { Criadouros } \\
\text { Commercial flocks }\end{array}$ & \multicolumn{4}{c}{$\begin{array}{c}\text { Idade (meses) } \\
\text { Age (months) }\end{array}$} \\
\cline { 2 - 5 } & 23 & 25 & 27 & 29 \\
\hline & & \multicolumn{2}{c}{$\begin{array}{c}\text { Intestino grosso vazio (\%) } \\
\text { Empty large gut (\%) }\end{array}$} \\
Faz. Vale da Serra & $17,37^{\mathrm{ab}}$ & $19,43^{\mathrm{bc}}$ & $22,84^{\mathrm{ab}}$ & $21,89^{\mathrm{b}}$ \\
Pró-Fauna & $17,33^{\mathrm{ab}}$ & $20,52^{\mathrm{bc}}$ & $24,55^{\mathrm{a}}$ & $22,02^{\mathrm{b}}$ \\
Agrotec & $15,94^{\mathrm{b}}$ & $21,52^{\mathrm{bc}}$ & $23,93^{\mathrm{a}}$ & $21,32^{\mathrm{b}}$ \\
Faz. São Romão & $21,94^{\mathrm{a}}$ & $18,16^{\mathrm{c}}$ & $20,74^{\mathrm{ab}}$ & $23,68^{\mathrm{b}}$ \\
Faz. Campo Redondo & $19,42^{\mathrm{ab}}$ & $24,04^{\mathrm{b}}$ & $21,51^{\mathrm{ab}}$ & $25,01^{\mathrm{ab}}$ \\
Faz. Lambari & $14,84^{\mathrm{b}}$ & $17,88^{\mathrm{c}}$ & $9,61^{\mathrm{c}}$ & $15,84^{\mathrm{c}}$ \\
Faz. Rios dos Bois & $17,51^{\mathrm{ab}}$ & $23,85^{\mathrm{b}}$ & $19,05^{\mathrm{b}}$ & $24,17^{\mathrm{a}}$ \\
Faz. Alcantilhado & $19,44^{\mathrm{ab}}$ & $29,72^{\mathrm{a}}$ & 20,75 & $29,78^{\mathrm{ab}}$ \\
Média (Mean) & 17,97 & 21,90 & 6,93 & 23,10 \\
CV(\%) & 9,79 & 10,11 & 7,87
\end{tabular}

Médias seguidas por letras iguais, na mesma coluna, não diferem entre si pelo teste Duncan $(P>0,05)$; CV= coeficiente de variação. Means indicated by the same letters, in the same column, do not differ by Duncan range test (P>.05); CV = coefficient of variation.

R. Bras. Zootec., v.32, n.1, p.10-18, 2003 
compartimento de capacidade relativa de conteúdos.

Nas Tabelas 6, 7 e 8 estão representados os comprimentos médios do TGI, dos intestinos delgadoe grosso, que apresentaram médias significativamente diferentes $(\mathrm{P}<0,05)$ entre os criadouros. O TGI cheio apresentou comprimento médio de $72,75 \mathrm{~cm}$, sendo encontradas maiores médias nos animais dos criadouros Vale da Serra $(95,05 \mathrm{~cm})$ e Pró-Fauna $(81,45 \mathrm{~cm})$, que tiveram os melhores desenvolvimentos.

Para o intestino delgado, foi obtida a média de $46,68 \mathrm{~cm}$ (Tabela 7) e para o intestino grosso, de $14 \mathrm{~cm}$ (Tabela 8). Com P. expansa, Moreira \& Loureiro (1992) descreveram que a relação entre o comprimento da carapaça e o comprimento total do TGI foi de $3,70 \%$, intestino delgado, $2,60 \%$, e para o intestino grosso de $0,78 \%$. Bjornal (1989), em discussão

Tabela 5 - Capacidade de armazenamento do estômago em relação ao TGI cheio de Podocnemis expansa, com idades entre 23 e 29 meses, provenientes de criadouros comerciais $(n=5)$

Table 5 - Stomachal storage capacty in relation to the full TGI of Podocnemis expansa from 23 to 29 months, from commercial flocks $(n=5)$

\begin{tabular}{|c|c|c|c|c|}
\hline \multirow[t]{2}{*}{$\begin{array}{l}\text { Criadouros } \\
\text { Commercial flocks }\end{array}$} & \multicolumn{4}{|c|}{$\begin{array}{l}\text { Idade (meses) } \\
\text { Age (months) }\end{array}$} \\
\hline & 23 & 25 & 27 & 29 \\
\hline & \multicolumn{4}{|c|}{$\begin{array}{c}\text { Capacidade de alimento no estômago (\%) } \\
\text { Stomachal storage capacity }(\%)\end{array}$} \\
\hline Faz. Vale da Serra & $73,04^{\mathrm{bc}}$ & $71,78^{\mathrm{ab}}$ & $57,53^{\mathrm{a}}$ & $75,56^{\mathrm{a}}$ \\
\hline Pró-Fauna & $93,03^{\mathrm{a}}$ & $61,90^{\mathrm{b}}$ & $68,30^{\mathrm{a}}$ & $70,15^{\mathrm{a}}$ \\
\hline Agrotec & $74,83^{\mathrm{abc}}$ & $80,08^{\mathrm{a}}$ & $82,23^{\mathrm{a}}$ & $75,94^{\mathrm{a}}$ \\
\hline Faz. São Romão & $64,34^{\mathrm{c}}$ & $69,01^{\mathrm{ab}}$ & $73,40^{\mathrm{a}}$ & $77,45^{\mathrm{a}}$ \\
\hline Faz. Campo Redondo & $77,61^{\mathrm{abc}}$ & $68,18^{\mathrm{b}}$ & $71,01^{\mathrm{a}}$ & $42,50^{b}$ \\
\hline Faz. Lambari & $60,89^{c}$ & $68,01^{\mathrm{b}}$ & $66,04^{\mathrm{a}}$ & $70,78^{a}$ \\
\hline Faz. Rios dos Bois & $82,22^{\mathrm{ab}}$ & $72,56^{\mathrm{ab}}$ & $66,91^{\mathrm{a}}$ & $45,59^{b}$ \\
\hline Faz. Alcantilhado & $62,27^{\mathrm{c}}$ & $70,74^{\mathrm{ab}}$ & $60,71^{\mathrm{a}}$ & $70,78^{a}$ \\
\hline Média (Mean) & 73,54 & 70,34 & 68,65 & 66,10 \\
\hline $\mathrm{CV}(\%)$ & 12,77 & 8,87 & 19,34 & 11,95 \\
\hline
\end{tabular}

Médias seguidas por letras iguais, na mesma coluna, não diferem entre si pelo teste Duncan $(P>0,05)$; $C V=$ coeficiente de variação. Means indicated by the same letters, in the same column, do not differ by Duncan range test ( $P>.05)$; $C V=$ coefficient of variation.

Tabela 6 - Comprimento médio $(\mathrm{cm})$ do trato gastrointestinal (TGI) cheio de Podocnemis expansa, com idades entre 23 e 29 meses, provenientes de criadouros comerciais $(n=5)$

Table 6 - Mean length of the full gastrointestinal tract (TGI) of Podocnemis expansa from 23 to 29 months, from commercial flocks $(n=5)$

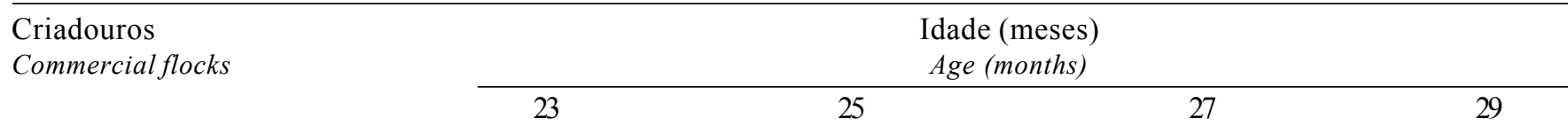

Comprimento do TGI cheio $(\mathrm{cm})$

Full TGI length (cm)

Faz. Vale da Serra

Pró-Fauna

Agrotec

Faz. São Romão

Faz. Campo Redondo

Faz. Lambari

Faz. Rios dos Bois

Faz. Alcantilhado

Média (Mean)

CV $(\%)$

$90,10^{\mathrm{a}}$
$74,50^{\mathrm{bc}}$
$77,90^{\mathrm{b}}$
$66,80^{\mathrm{cd}}$
$67,80^{\mathrm{cd}}$
$64,30^{\mathrm{d}}$
$65,90^{\mathrm{d}}$
$60,20^{\mathrm{d}}$
70,93
8,62
$100,53^{\mathrm{a}}$

$86,60^{\mathrm{b}}$

$75,49^{\mathrm{c}}$

$87,60^{\text {b }}$

$75,40^{\mathrm{c}}$

$67,58^{\text {cd }}$

$61,20^{\text {de }}$

$57,20^{\mathrm{e}}$

76,45

8,53

$97,37^{\mathrm{a}}$
$88,60^{\mathrm{b}}$
$66,40^{\mathrm{e}}$
$72,70^{\mathrm{e}}$
$81,00^{\mathrm{c}}$
$73,00^{\mathrm{de}}$
$72,60^{\mathrm{e}}$
$48,40^{\mathrm{f}}$
74,43
7,41

$92,20^{\mathrm{a}}$

$76,10^{\mathrm{b}}$

$66,40^{\text {cd }}$

$71,46^{\text {bc }}$

$70,70^{\text {bcd }}$

$62,50^{\mathrm{d}}$

$63,20^{\text {cd }}$

$51,00^{\mathrm{e}}$

69,19

9,01

Médias seguidas por letras iguais, na mesma coluna, não diferem entre si pelo teste Duncan $(P>0,05)$; CV = coeficiente de variação. Means indicated by the same letters, in the same column, do not differ by Duncan range test $(P>.05) ; C V=$ coefficient of variation. 
Chelonia mydas (tartaruga-verde). Esse herbívoro se especializou em dietas folívoras e depende da microflora simbiótica do intestino grosso, que possui um tamanho em torno de três vezes o tamanho do intestino delgado. $\mathrm{O}$ autor citado enfatiza que os herbívoros consumidores oportunistas, que ingerem alimentos variados, deveriam ter a mais ampla variação na resposta digestória.

\section{Conclusões}

As análises das relações corporais indicaram que o estômago e o intestino delgado apresentam as maiores capacidades de armazenamento, sugerindo que essas vísceras desempenham importante função na digestão de alimentos consumidos por $P$. expansa jovens, em cativeiro.

\section{Agradecimento}

À Associação PRÓ-TARTARUGA, pelo apoio financeiro concedido para a publicação deste artigo.

\section{Literatura Citada}

ALFINITO, J. A tartaruga verdadeira do amazonas: sua criação. Belém: Faculdade de Ciências Agrárias do Pará, 1980. 69p. Informe Técnico, 5.

ALMEIDA, S.S.; SÁ, P.G.; GARCIA, A. Vegetais utilizados como alimento por Podocnemis (Chelonia) na região do baixo Rio Xingu (Brasil/Pará). Boletim do Museu Paraense Emílio Goeldi-Botânica, v.2, n.2, p.199-211, 1986.

ANDRADE, M.L. Efeitos das relações energia: proteína e aminoácidos sulfurados e lisina na ração pré-inicial sobre o desempenho de frangos de corte. Goiânia: Universidade Federal de Goiás, 2000. 29p. Monografia (Especialização em Zootecnia) - Universidade Federal de Goiás, 2000.

BJORNAL, K. Flexibility of digestive responses in two generalist herbivores, the tortoises Geochelone carbonaria and Geochelone denticulata. Oecologia, v.78, p.317-321, 1989.

CENAQUA. Considerações biológicas sobre os quelônios. Pimenteiras/RO: Centro Nacional dos Quelônios da Amazônia-Ibama. 1992. 45p. Relatório de Atividades.

CENAQUA. Conservação e criação comercial de quelônios. Goiânia: Centro Nacional dos Quelônios da Amazônia- Ibama. 1992. 34p. Apostila.
CENAQUA. Atividades da área de criação em cativeiro no exercício de 2000. Goiânia: Centro Nacional dos Quelônios da Amazônia. IBAMA, 2000. 20p. Relatório.

LIMA, M.G.H.S. A importância das proteínas de origem animal e vegetal no primeiro ano de vida da tartarugada-amazônia - Podocnemis expansa (Schweigger, 1812). Manaus: Universidade do Amazonas e Instituto Nacional de Pesquisa da Amazônia, 1998. 93p. Dissertação (Mestrado em Ciência de Alimentos) - Universidade do Amazonas e Instituto Nacional de Pesquisa da Amazônia, 1998.

MOREIRA, G.R.S.; LOUREIRO, J.S. Contribucion al estudio de la morfologia del tracto digestivo de individuos jovens de Podocnemis expansa (Testudinata: Pelomedusidae). Acta Zoologica Iliboana, v.41, p.345-348, 1992.

OJASTI, J. La tortuga arrau del Orinoco. Um recurso impropiamente utilizado. Defensa de la Naturaleza, v.2, p.3-9, 1971.

OLIVEIRA, G.M.; SANTOS, E.E.D., LUZ, V.L.F. Estudo morfológico do tubo digestivo de Podocnemis expansa (tartaruga-da-amazônia). In: CONGRESSO BRASILEIRO DE ZOOLOGIA, 21., 1996, Santa Maria. Resumos... Santa Maria: Sociedade Brasileira de Zoologia/Universidade Federal do Rio Grande do Sul, 1996. n.190, [s.p].

RAN. Atividades da área de criação em cativeiro no exercício de 2001. Goiânia: Centro de Conservação e Manejo de Répteis e Anfíbios. Ibama, 2001. 23p. Relatório.

SAMPAIO, I.B.M. Estatística aplicada à experimentação animal. Belo Horizonte: Fundação de Ensino e Pesquisa em Medicina Veterinária e Zootecnia, Universidade Federal de Minas Gerais, 1998. 221p.

SANTOS, A.L.Q.; BELETTI, M.E.; QUEIROZ, R.P. et al. Estudo morfológico do tubo digestivo da tartaruga-daamazônia Podocnemis expansa. Uberlândia: Universidade Federal de Uberlândia, 1998. 15p. Relatório.

SILVA NETO, P.B. Abate de tartarugas-da-amazônia. São Paulo: Pró-Fauna Assessoria e Comércio Ltda. Convênio Empresa Pró-Fauna e Cenaqua-Ibama, 1998. 48p. Relatório.

TERÁN, A.F. Alimentação de cinco espécies de quelônios em Costa Marques, Rondônia, Brasil. Manaus: Instituto Nacional de Pesquisas da Amazônia, Fundação Universidade do Amazonas, 1992. 65p. Dissertação (Mestrado em Ciências Biológicas) - Instituto Nacional de Pesquisas da Amazônia, Fundação Universidade do Amazonas, 1992.

TERÁN, A.F.; VOGT, R.C.; GOMEZ, M.F.S. Food habits of na assemblage of five species of turtles in Rio Guaporé, Rondônia, Brazil. Journal of Herpetology, v.29, n.4, p.536-547, 1995.

Recebido em: 29/06/01 Aceito em: 16/04/02 